\title{
A Short Survey and Open Questions on Compact $\mathcal{Q}$-Groups
}

\section{Francesco G. Russo}

Department of Mathematics and Applied Mathematics, University of Cape Town, Private Bag X1, Rondebosch, Cape Town 7701, South Africa; francescog.russo@yahoo.com

Received: 17 October 2019; Accepted: 8 November 2019; Published: 13 November 2019

\begin{abstract}
Finite $\mathcal{Q}$-groups have been recently studied and form a class of solvable groups, which satisfy interesting structural conditions. We survey some of their main properties and introduce the idea of $\mathcal{Q}$-group for compact $p$-groups ( $p$ prime). A list of open questions is presented, along with several connections of arithmetic nature on a problem originally due to Frobenius.
\end{abstract}

Keywords: local breadth; compact p-groups; Euler's function; Haar measure

\section{Global Breadth and Local Breadth of Finite Groups}

The first examples of finite groups, where one needs to distinguish between the exponent and the order, come to our attention when we look at the Vierergruppe $V=C_{2} \times C_{2}$ of Klein [1] and at the cyclic group $C_{4}=\mathbb{Z}(4)$ of order four. Both of them are groups of order four, but $V$ has no elements of order four, while $C_{4}$ has one element of order four. The exponent $\exp (G)=e$ of a finite group $G$ is the smallest positive integer such that $g^{e}=1$ for all $g \in G$, and this notion helps to justify the absence of elements of order four in $V$, even if $|V|=4$. Frobenius [2,3] worked on the following problem long time ago.

Problem 1 (Frobenius Problem, 1895). Given a finite group $G$ and $m \mid \exp (G)$, define

$$
L_{m}(G)=\left\{x \in G: x^{m}=1\right\}
$$

and study relations between $m,\left|L_{m}(G)\right|$ and the structure of $G$.

Frobenius showed that a divisor $m$ of $|G|$ divides also $\left|L_{m}(G)\right|$, that is,

$$
\left|L_{m}(G)\right|=m k \text { for some } k \geq 1 \text {, }
$$

on the other hand, he conjectured that

Conjecture 1 (Frobenius' Conjecture, 1903). For $k=1$, the $m$ elements of $L_{m}(G)$ form a characteristic subgroup in any finite group $G$.

This was indeed shown by liyori and Yamaki in [4-8] with the help of the classification of finite simple groups. The elegance of the proof of Frobenius' Conjecture cannot be summarized here, but its importance is clear if we think that no restriction on $G$ is given (unless the fact that it is finite).

A more recent problem, studied in [9-14], involves the growth of $k$ and corresponding structural conditions on a finite group $G$. The authors of [9-15] studied the local breadth of a finite group $G$, that is,

$$
\mathbf{b}_{m}(G)=\left|L_{m}(G)\right| \cdot m^{-1}
$$


which represents a numerical quantity of normalization of $\left|L_{m}(G)\right|$ via $m$. In particular, one can introduce the function

$$
f: m \in \mathbb{N} \mapsto f(m)=\mathbf{b}_{m}(G) \in \mathbb{N}
$$

depending only on $m$ and study groups where $f(m)$ grows very slowly. It is also good to mention the notion of global breadth, introduced in [11,12], that is, the maximum value of all the local breadths

$$
\mathbf{B}(G)=\max \left\{\mathbf{b}_{m}(G): m \mid \exp (G)\right\}
$$

which are present in a finite group G. Roughly speaking, this represents a numerical quantity of optimisation for the local breadths of a finite group.

Problem 2 (Inverse Problem to Frobenius' Theorem, see [13]). Classify all finite groups $G$ such that $\left|L_{m}(G)\right| \leq \psi(m)$ for all $m \mid \exp (G)$, where $\psi: m \in \mathbb{N} \mapsto \psi(m) \in \mathbb{N}$ is an arithmetic function (depending only on $m$ ).

A first body of results was due to Meng and Shi [13] in this line of research. They described finite groups with $f(m) \leq 2$.

Theorem 1 (See [13], Main Theorem and [11], list in §4). A finite group $G$ with $m \mid \exp (G)$ has $f(m) \leq 2$ if and only if one of the following conditions holds:

(i) $\mathbb{Z}(n)$ for some $n \geq 1$;

(ii) $\mathbb{Z}(h) \times \mathbb{Z}\left(2^{k-1}\right) \times \mathbb{Z}(2)$ with $h$ odd and $k \geq 2$;

(iii) $\mathbb{Z}(h) \times Q_{8}$ with $h$ odd and

$$
Q_{8}=\left\langle a, b: a^{4}=1, a^{2}=b^{2}, b^{-1} a b=a^{-1}\right\rangle
$$

quaternion group of order 8 ;

(iv) $\mathbb{Z}(h) \times Q D_{2^{t}}$ with $h$ odd, $t \geq 4$ and

$$
Q D_{2^{t}}=\left\langle a, b: a^{2^{t-1}}=b^{2}=1, b^{-1} a b=a^{1+2^{t-2}}\right\rangle
$$

quasi-dihedral 2-group of order $2^{t}$;

(v) $\mathbb{Z}(h) \times D$ with $h$ odd, $s \geq 1, \operatorname{gcd}(h, 6)=1$ and

$$
D=\left\langle a, b: a^{3}=b^{2^{s}}=1, b^{-1} a b=a^{-1}\right\rangle
$$

nilpotent metacyclic of order $3 \cdot 2^{s}$.

In particular, $G$ is a nilpotent group in each of the five cases above.

A finite group $G$ is metacyclic if it is extension of a cyclic group by a cyclic group, that is, if $G=A B$ for some cyclic normal subgroup $A$ of $G$ such that $G / A \simeq B$ is cyclic. Quasi-dihedral 2-groups can be found in ([16] Pages 90-94) and are metacyclic. About metacyclic p-groups, see ([16] Kapitel III, §11): they were introduced by Zassenhaus in [17]. The reader has probably noted in (i) of Theorem 1 that we used the notation $\mathbb{Z}(n)$ for a cyclic group of order $n$, according to $[18,19]$. This is indeed more convenient when we will deal with compact groups, where the symbol $\mathbb{Z}_{p}$ denotes the additive group of $p$-adic integers ([19] Example E1.10). Chen, Meng and Shi ([9] [Theorems 1.1, 1.2) improved the classification based on the bound $f(m) \leq 2$ in Theorem 1 with another one, based on the upper bound $f(m) \leq 3$. Successive generalizations have been found and the reader can refer to $[9,14,15]$. Theorem 1 may be reformulated via the global breadth in the following way: 
Theorem 2 (See [12]). A finite group $G$ has $\mathbf{B}(G) \leq 2$ if and only if it satisfies one of the conditions of Theorem 1.

In Theorems 1 and 2, we have finite groups with local breadth and global breadth at most two, but ([11] Example 2.10) shows more general contexts where we can find different values of local breadths and global breadths. The local breadth is indeed dependent on the specific divisor of the exponent of the group, while the global breadth is not depending on it and describes a behaviour of growth of (2) and of (3) for the whole group. The notion of finite $\mathcal{Q}$-group was introduced in [10], in order to classify finite groups with slow growth of (3):

Definition 1 (See [10], P. 1962). A finite group $G$ is called $\mathcal{Q}$-group, if $f(m) \leq m$ with $m \mid \exp (G)$, where $f(m)$ is the function introduced in (3).

We can also interpret Definition 1 noting that it describes finite groups with local breadth which is bounded by a quadratic function of the divisor of the exponent. Corresponding classifications are presented in ([10] Theorems 3.2, 3.5, 3.6, 3.8, 3.12, 3.14), which we are going to summarize below.

Before doing this, we need to recall from ([16] Kapitel IV, §4) that a normal p-complement of a finite group $G$ for a prime $p$ is a normal subgroup $N$ of $G$ of order coprime to $p$ and index a power of $p$. In this situation we have that $G=N \rtimes H$ is the semidirect product of $N$ by $H$ with $\operatorname{gcd}(|N|,|H|)=1$ and we briefly say that $G$ is $p$-nilpotent, that is, if $G$ possesses a normal $p$-complement. Classical results of Schur and Zassenhaus ([16] Kapitel I, Hauptsatz 18.1, 18.2, 18.3) describe the presence of complements in finite groups, but the situation is very different in the infinite case and ([18] §2.3) can give an idea of what happens for locally compact groups. Before to state the following result, we recall that $F(G)$ denotes the Fitting subgroup of finite group $G$, that is, the largest nilpotent normal subgroup of $G$.

Theorem 3 (Structure of Finite $\mathcal{Q}$-groups, see [10]). Let $G$ be a finite group, $m \mid \exp (G)$ and $f(m) \leq$ m. Then

(i) $G$ is solvable;

(ii) $G$ has a 2-nilpotent normal subgroup $M$ of index $|G: M| \in\{1,3\}$;

(iii) $G$ is the product of two cyclic groups with trivial intersection, provided $G$ is a p-group with $p$ odd prime;

(iv) $G$ is metabelian and $G / F(G)$ is cyclic, provided $\operatorname{gcd}(|G|, 6)=1$;

(v) There exists a solvable nonmetabelian nonnilpotent $\mathcal{Q}$-group $G$ such that $\operatorname{gcd}(|G|, 6) \neq 1$.

One can see in [10] that the example for the condition (v) of Theorem 3 is given by the special linear group $\operatorname{SL}(2,3)$ on a field with 3 elements and the relevance of this condition is due to the fact that one could ask whether the assumption $\operatorname{gcd}(|G|, 6)=1$ can be removed in (iv) of Theorem 3 or not. The consideration of $G=\operatorname{SL}(2,3)$ answers negatively, showing that finite solvable nonmetabelian nonnilpotent $\mathcal{Q}$-groups may be produced with linear groups.

We also note that (ii) of Theorem 3 is a result of decomposition in semidirect product. In fact (ii) of Theorem 3 may be rephrased by the presence of a decomposition of the form $G=M \rtimes C$ for some finite normal 2-subgroup $M$ such that $G / M$ is either trivial or cyclic of order three. Similarly (iv) of Theorem 3 gives another structural condition, since it shows that $F(G)$ is abelian (see [10] for details) and $G$ can be decomposed in the product $G=F(G) C$ for a cyclic group $C \simeq G / F(G)$. We find again a semidirect product $G=F(G) \rtimes C$ when $\operatorname{gcd}(|C|,|F(G)|)=1$, so there is another structural result, originating from arithmetic restrictions on the local breadth. Our notations are standard and can be found in [19-24]. 


\section{Compact $\mathcal{Q}$-Groups}

According to the terminology of $[18,19,21]$, it is good to point out that a compact (Hausdorff) $p$-group and a pro- $p$-group are exactly the same object. This means that a compact $p$-group can be written as projective limit of finite $p$-groups;

$$
G=\lim _{N \in \mathcal{N}(G)} G / N, \text { where } \mathcal{N}(G)=\{N=\bar{N} \triangleleft G: G / N \text { is a finite } p-\text { group }\}
$$

and in particular $\mathcal{N}(G)$ is a filter basis for the topology of $G$. Note that open subgroups in $G$ are exactly those subgroups of index a power of $p$.

Compact $p$-groups constitute a natural framework, where we may extend Definition 1 . In order to make this, we recall from ([21] Definition 1.2.5) that it is possible to define for each integer $i \geq 0$ the $i$ th omega set $\Omega_{\{i\}}(G)$ and the $i$ th omega subgroup $\Omega_{i}(G)$ of a finite $p$-group $G$ by

$$
\Omega_{\{i\}}(G)=\left\{x \in G: x^{p^{i}}=1\right\} \text { and } \Omega_{i}(G)=\left\langle\Omega_{\{i\}}(G)\right\rangle .
$$

Dually we may also define the $i$ th agemo set $\mho_{\{i\}}(G)$ and the $i$ th agemo subgroup $\mho_{i}(G)$ of a finite p-group $G$ by

$$
\mho_{\{i\}}(G)=\left\{x^{p^{i}}: x \in G\right\} \text { and } \mho_{i}(G)=\left\langle\mho_{\{i\}}(G)\right\rangle .
$$

However, the consideration of the dihedral 2-group $D_{8}$ of order 8 shows that $\Omega_{1}\left(D_{8}\right)=D_{8}$ and $\Omega_{\{1\}}\left(D_{8}\right) \neq D_{8}$, so the inclusion $\Omega_{\{i\}}(G) \subseteq \Omega_{i}(G)$ can be strict in general. Further examples can be given, in order to show that even the inclusion $\mho_{\{i\}}(G) \subseteq \mho_{i}(G)$ can be strict in general.

According to ([21] Definition 1.2.9 and Lemma 1.2.10) and ([16] Kapitel III, §10), we may focus on certain $p$-groups introduced by Hall in 1933; a finite $p$-group $G$ is regular if for all $x, y \in G$ and any integer $i>0$ the following identity

$$
(x y)^{p^{i}}=x^{p^{i}} y^{p^{i}} s^{p^{i}} \text { for some } s \in \gamma_{2}(\langle x, y\rangle)=[\langle x, y\rangle,\langle x, y\rangle]
$$

is satisfied, where $\gamma_{2}(G)=[G, G]$ denotes the well known 2nd term of the lower central series of $G$. We report some elementary facts from ([21] [Lemmas 1.2.11, 1.2.12 and 1.2.13).

Lemma 1 (See [21]). If $G$ is a finite regular p-group, then

$$
\Omega_{\{i\}}(G)=\Omega_{i}(G), \mho_{\{i\}}(G)=\mho_{i}(G), G / \Omega_{i}(G) \simeq \mho_{i}(G), G / \mho_{i}(G) \simeq \Omega_{i}(G) .
$$

In particular, this happens if $G$ is a finite abelian p-group.

A finite $p$-group $G$ is powerf $u l$ if $\gamma_{2}(G) \subseteq \mho_{1}(G)$ for odd $p$, or if $\gamma_{2}(G) \subseteq \mho_{2}(G)$ for $p=2$. This is equivalent to require $G / \mho_{1}(G)$ abelian for odd $p$, or $G / \mho_{2}(G)$ abelian for $p=2$. Clearly, finite abelian $p$-groups are both powerful $p$-groups and regular $p$-groups, but there are finite nonregular powerful $p$-groups (see [25] Pages 497-498). Powerful $p$-groups were introduced by Lubotzky and Mann [25,26] and satisfy similar properties to Lemma 1 (see also [22,27]). A result from [28] shows this behaviour.

Lemma 2 (See [28]). Assume $G$ is a finite powerful p-group. Then

$$
\left|G: \mho_{i}(G)\right|=\left|\Omega_{\{i\}}(G)\right| \text { and }\left|G: \Omega_{i}(G)\right|=\left|\mho_{\{i\}}(G)\right| .
$$

Using the terminology of the previous section, we have the following fact, which is clearly an application of Lemmas 1 and 2. 
Lemma 3. For a finite powerful p-group $G$ and positive integer $m \leq \log _{p}(\exp (G))$,

$$
\mathbf{b}_{p^{m}}(G)=\left|G: \mho_{m}(G)\right| \cdot p^{-m}
$$

The same result is true if $G$ is a regular finite p-group, or a finite abelian p-group.

A compact $p$-group $G$ admits a left invariant Haar measure $\mu$, which is a positive Radon measure on a $\sigma$-algebra containing Borel sets (see [19] Theorem 2.8). The support of $\mu$ is bounded, due to the compactness of $G$, so we may look at $G$ as a measure space with a unique normalized measure $\mu$. From the fact that $\mu$ is monotonically increasing, if $H$ is closed subgroup of $G$, then $\mu(H) \leq \mu(G)=1$. Note also that for any positive integer $k$, one has immediately

$$
\mu(H)= \begin{cases}\frac{1}{k}, & \text { if }|G: H|=k \\ 0, & \text { if }|G: H|=\infty\end{cases}
$$

Another ingredient, which we need to have, is the notion of exponent for compact $p$-groups. From (5), a compact $p$-group $G=\lim _{N \in \mathcal{N}(G)} G / N$ has exponent

$$
\exp (G) \begin{cases}\text { finite (equal to } \left.p^{e}\right), & \text { if } \lim _{N \in \mathcal{N}(G)} \exp (G / N)=p^{e} \in \mathbb{N} \\ \text { non-finite, } & \text { otherwise. }\end{cases}
$$

This concept gives the usual notion of exponent, when $G$ is a finite $p$-group. Note that classical results of Kulikov, Prüfer and Baer describe abelian groups which are bounded, see ([29] 4.3.5, 4.3.6, $4.3 .14,4.3 .15,4.3 .16)$, and these have finite exponent according to (10). The group of $p$-adic integers $\mathbb{Z}_{p}$ is a compact abelian $p$-group of infinite exponent and is torsion-free. Another compact abelian $p$-group of infinite exponent is the Prüfer group $\mathbb{Z}\left(p^{\infty}\right)$. This has no nontrivial torsion-free elements. Then we come to the direct sum of cyclic groups $\mathbb{Z}(p)$, which has finite exponent equal to $p$ and no nontrivial torsion-free elements. Of course, the situation changes drastically from what we observed in finite $p$-groups.

Definition 2. The local breadth $\mathbf{b}_{p^{m}}(G)$ of a compact p-group $G$ of finite exponent $\exp (G)$ and $m \leq$ $\log _{p}(\exp (G))$ is the non-negative integer

$$
\mathbf{b}_{p^{m}}(G)=\mu\left(\overline{\mho_{m}(G)}\right)^{-1} \cdot p^{-m}
$$

where $\mu$ is the unique normalized left invariant Haar measure on G.

The previous discussion shows that Definition 2 is well posed. Moreover an application of the definitions, (9) and Lemmas 1-3 explains the following formula for the local breadth of a compact p-group.

Lemma 4. If $G$ is a compact p-group $G$ of finite exponent with $m \leq \log _{p}(\exp (G))$, then

$$
\mathbf{b}_{p^{m}}(G)=\left|G: \overline{\mho_{m}(G)}\right| \cdot p^{-m}
$$

and $\mathbf{b}_{p^{m}}(G)$ is a positive integer if and only if $\overline{\mho_{m}(G)}$ is open. In particular, if $G$ is a finite powerful p-group, or a finite abelian p-group, or a finite regular p-group, then $\mathbf{b}_{p^{m}}(G)$ is exactly (2).

We may generalize the abelian cases of Theorem 1 in the following way. 
Theorem 4. Assume $G$ is a compact abelian p-group of finite exponent with $m \leq \log _{p}(\exp (G))$. If $1=$ $\mathbf{b}_{p^{m}}(G)$, then $G$ is bounded for any choice of $p$. If $\mathbf{b}_{p^{m}}(G)=2$, then $p=2$ and $G$ is a bounded 2-group.

Proof. If $\mathbf{b}_{p^{m}}(G)=1$, then $\mu\left(\widetilde{\mho}(G)^{-1}=p^{m}\right.$. Lemma 1 is valid also for infinite abelian $p$-groups, so Lemma 4 implies for all $m$ that $\left|G: \overline{\mho_{m}(G)}\right|=\left|\overline{\Omega_{m}(G)}\right|=p^{m}$. Denoting $\exp (G)=p^{e}=h$ for some $h \in \mathbb{N}$, we have $G=\overline{\Omega_{h}(G)} \supseteq \overline{\Omega_{h-1}(G)} \supseteq \ldots \supseteq \overline{\Omega_{2}(G)} \supseteq \overline{\Omega_{1}(G)} \supseteq \overline{\Omega_{0}(G)}=1$, so in particular $m=h$ shows that $G$ is a bounded abelian $p$-group. Assume now $\mathbf{b}_{m}(G)=2$. If $p=2$, then the same argument shows that $\mu\left(\overline{\mho_{m}(G)}\right)^{-1}=2 \cdot 2^{m}$ and $G$ must be a bounded 2-group. If $p \neq 2$, then $\overline{\mho_{m}(G)}$ must be open by Lemma 4 and simultaneously $\mu\left(\overline{\mho_{m}(G)}\right)^{-1}=\left|G: \overline{\mho_{m}(G)}\right|=2 \cdot p^{m}$ is not a prime power, which is impossible. The result follows.

A series of open problems arise naturally at this point. First of all, it is meaningful to introduce the following notion.

Definition 3. A compact p-group $G$ of finite exponent is a $\mathcal{Q}$-group, if $1 \leq \mathbf{b}_{p^{m}}(G) \leq p^{m}$, for any choice of $m \leq \log _{p}(\exp (G))$.

Note that Theorem 4 explains what happens in case of compact $p$-groups which are abelian and it provides a first generalization of the abelian cases which we encounter in Theorem 1 , so this gives significant evidences. A successive step might be the following.

Problem 3. Use Lemmas 1-4 as models to generalize to nonabelian compact $\mathcal{Q}$-groups within the class of compact p-groups of finite exponent.

The class of powerful pro- $p$-groups contains indeed the class of compact abelian $p$-groups of finite exponent, so it is a meaningful class of compact groups where one can investigate $\mathcal{Q}$-groups. Let's now recall from $[19,24]$ that it is possible to consider a profinite version of the notion of Fitting subgroup. In fact one can define the Fitting subgroup $F(G)$ of a profinite group $G$ as the largest normal closed nilpotent subgroup of $G$. Note that $p$-nilpotent profinite groups are described in $[30,31]$ with an appropriate discussion of the complements.

Problem 4. Consider a nonabelian compact p-group $G$ of finite exponent with Fitting subgroup $F(G)$. If $G$ is $\mathcal{Q}$-group, then $G / F(G)$ might be procyclic, generalizing the evidence of the finite case in Theorem 3 (iv). Moreover, it is reasonable to expect that there are no nonsolvable compact p-groups which are $\mathcal{Q}$-groups, generalizing Theorem 3 (i).

To this end it would be natural to proceed in the following way:

(i) Develop a notion of local breadth for a class of compact groups containing compact $p$-groups;

(ii) Work toward a formulation of local breadth in a way that does not explicitly stipulate/reference a finite exponent hypothesis;

(iii) Determine whether locally compact ablelian $p$-groups of approximately finite exponent (see [18] Definition 3.30) constitute a significant generalization for this effort, or not.

Of course, the main idea would be to use the Haar measure to facilitate a parallel to the proportional behaviour exhibited in the finite setting. On the other hand, Definition 3 is designed for compact $p$-groups of finite exponent, so it does not work for instance for compact Lie groups. 


\section{Connections with the Euler's Function}

Chronologically [32] appears two years after [10], but even [33-35] do not mention the so called "Inverse Problem to Frobenius' Theorem" in the study of structural properties of groups by restrictions of numerical nature. This gives two parallel approaches to the classification of finite groups via the size of their local breadth which we can briefly illustrate here via a relevant observation.

Let us change completely perspective and look numerically at the size of $L_{m}(G)$ when $G$ is a finite group. Consider the Euler function $\varphi(k)$ and define the number

$$
c_{k}=\mid\{C \leq G: C \text { is a cyclic subgroup of order } k\} \mid
$$

of cyclic subgroups of $G$ of order exactly $k$. Having in mind (1), one can see that

$$
\left|L_{m}(G)\right|=\sum_{k \mid m} c_{k} \varphi(k)=m \cdot f(m),
$$

where $f(m) \geq 1$ is an integer depending only on $m$ and $G$. In fact $f(m)$ in (11) coincides with (3), that is, it is the local breadth of $G$ in Definition 2. Involving an approach with the Euler function, a first important problem is the following.

Problem 5. Generalize (11) to compact p-groups and study its influence on the group structure.

From Definitions 3, we can firstly consider a compact $p$-group $G$ which is powerful and for this we have a natural replacement for the local breadth, as seen in Lemma 4 . So the equality " $\left|L_{m}(G)\right|=m \cdot f(m)$ " can be formalized without problems for compact $p$-groups which are powerful. On the other hand, the corresponding continuous meaning of the sum

$$
\sum_{k \mid m} c_{k} \varphi(k)
$$

should be properly justified and this seems to be difficult to formalize.

Let's come back to the case of finite groups and investigate another interesting aspect of finite $\mathcal{Q}$-groups. Note that the Möbius function $\lambda$ can be defined as

$$
\lambda: i \in \mathbb{N} \mapsto \lambda(i)= \begin{cases}0 & \text { if } i=u^{2} v \text { for some } u, v \in \mathbb{N} \text { and } u \neq 1 \\ 1, & \text { if } i=p_{1} \ldots p_{2 k} \text { for some } p_{1}, \ldots, p_{2 k} \in \mathbb{P} \\ -1, & \text { if } i=p_{1} \ldots p_{2 k-1} \text { for some } p_{1}, \ldots, p_{2 k-1} \in \mathbb{P}\end{cases}
$$

In other words, $\lambda$ is the arithmetic function sending $i$ to 0 , if $i$ is divisible by a square different from 1 ; sending $i$ to 1 , if $i$ is a product of an even number of distinct primes; sending $i$ to -1 , if $i$ is a product of an odd number of distinct primes. The set of all prime numbers has been denoted by $\mathbb{P}$ in (12).

If $G$ is finite of order $|G|=n$ and $r, s$ two real numbers, it has been introduced the function

$$
T_{G}(r, s)=\sum_{k \mid n} g_{k, n / k}^{r, s} k(f(k)-1),
$$

in ([32] Page 683, §2.4) where $g_{k, n / k}^{r, s}$ are the coefficient expressed by the expansion ([32] (1) of Lemma 7) and $f(k)$ is exactly (3), that is, the local breadth of $G$. Different expressions are possible for (13) but we will focus on (13) above. The coefficients $g_{k, n / k}^{r, s}$ turn out to be non-negative and the case $g_{k, n / k}^{r, s}=0$ is completely described by ([32] Lemma 7 (1)), which we report below for sake of completeness. 
Lemma 5 (See [32], Lemma 7). For $j, m \in \mathbb{N}$ and $r, s$ two real numbers such that $s \leq \min \{0, r\}$, we define

$$
g_{m, j}^{r, s}=\sum_{i \mid j} \frac{\lambda(i)(m i)^{s}}{\varphi(m i)^{r}}
$$

where $\lambda$ is the Moebius function in (12). Then

$$
g_{m, j}^{r, s}=\frac{m^{s}}{\varphi(m)^{r}} \cdot \prod_{t=1}^{l}\left(1-p_{t}^{s-r}\right) \cdot \prod_{t=l+1}^{k}\left(1-\frac{p_{t}^{s}}{\left(p_{t}-1\right)^{r}}\right),
$$

where the prime factorization of $j$ is $j=p_{1}^{t_{1}} \ldots p_{k}^{t_{k}}$ and that of $m$ is given by $m=m^{\prime} p_{1}^{\alpha_{1}} \ldots p_{l}^{\alpha_{l}}$ with $l \leq k$ and $\operatorname{gcd}\left(m^{\prime}, j\right)=1$. In particular the coefficients $g_{m, j}^{r, s}$ are always positive and become zero if and only if one of the following holds:

(i) $s=r=0$ and $j \neq 1$;

(ii) $s=r, r \neq 0$ and $l \geq 1$;

(iii) $s=0, r \neq 0$ and $p_{t}=2$ for some $t \in\{l+1, \ldots, k\}$.

Restrictions on $T_{G}(r, s)$ allows us to detect nilpotency in $G$ by ([32] Theorem 5) and further properties were also noted in [33-35]. In particular, ([32] Questions 1.4) asks whether the equality $T_{G}(r, s)=0$ detects solvability for a finite group $G$ or not for suitable $(r, s)$, while ([32] Questions 1.5) asks whether the equality $T_{G}(r, s)=0$ implies structural properties for $G$ for suitable $(r, s) \in \mathbb{R}^{2}$ or not.

Thanks to Theorem 3, we are in the position to show that a large class of finite solvable groups, not necessarily nilpotent, satisfies the equation $T_{G}(r, s)=0$.

Theorem 5. If $(r, s) \in \mathbb{R}^{2}$ satisfy $1 \leq g_{k, n / k}^{r, s}$ and $f(k) \leq\left(g_{k, n / k}^{r, s}\right)^{-\frac{1}{2}} \leq k$ for all $k$ divisors of $n$, then $T_{G}(r, s)=0$ and $G$ is solvable.

Proof. It is useful to note that (13) becomes equal to zero when $f(k)=1$ and $f(k)=1$ happens if and only if $G$ satisfies (i) of Theorem 1 , that is, $G$ is cyclic. Therefore the real issue of studying $T_{G}(r, s)=0$ is when $f(k) \geq 2$, so there is no loss of generality in assuming $f(k) \geq 2$. Since all $g_{k, n}^{r, s} \geq 1$, we have $T_{G}(r, s) \geq 0$ and it is enough to show $T_{G}(r, s) \leq 0$ in order to conclude that $T_{G}(r, s)=0$. Now

$$
f(k) \leq\left(g_{k, n / k}^{r, s}\right)^{-\frac{1}{2}} \Rightarrow g_{k, n / k}^{r, s} f(k) \leq \sqrt{g_{k, n / k}^{r, s}} \Rightarrow \sum_{k \mid n} g_{k, n / k}^{r, s} k(f(k)-1) \leq 0
$$

which gives what we claimed $T_{G}(r, s)=0$. On the other hand, $G$ is a $\mathcal{Q}$-group because $f(k) \leq k$ and so it is solvable by (i) of Theorem 3. The result follows.

In particular, we deduce that:

Corollary 1. There exist finite $\mathcal{Q}$-groups satisfying the equation $T_{G}(r, s)=0$.

While the existence of $(r, s)$ is shown under the conditions of Theorem 5 , the structural conditions of $G$ are elucidated in Theorem 3. Again the infinite case suggests a new area of research:

Problem 6. Generalize Theorem 5 to compact p-groups and study when $T_{G}(r, s)=0$.

The replacement of the term $k(f(k)-1)$ in (13) may be done via the notion of local breath we gave in Definition 2 so the real issue is to have a corresponding formulation of Lemma 5 in case of compact $p$-groups. 
Funding: This research received no external funding.

Acknowledgments: I want to thank the referees and NRF for ISARP 2018-2020.

Conflicts of Interest: The authors declare no conflict of interest.

\section{References}

1. Klein, F. Vorlesungen über das Ikosaeder und die Auflösung der Gleichungen vom fünften Grade; Teubner: Leipzig, Germany, 1884.

2. Frobenius, G. Verallgemeinerung des Sylowschen Satzen. Berliner Sitz. 1895, 981-993.

3. Frobenius, G. Über einen Fundamentalsatz der Gruppentheorie. Berliner Sitz. 1903, 987-991.

4. Iiyori, N.; Yamaki, H. On a conjecture of Frobenius. Bull. Am. Math. Soc. 1991, 25, 413-416. [CrossRef]

5. Yamaki, H. A conjecture of Frobenius and the sporadic simple groups I. Commun. Algebra 1983, 11, $2513-2518$. [CrossRef]

6. Yamaki, H. A conjecture of Frobenius and the simple groups of Lie type I. Arch. Math. 1984, 42, $344-347$. [CrossRef]

7. Yamaki, H. A conjecture of Frobenius and the simple groups of Lie type II. J. Algebra 1985, 96, 391-396. [CrossRef]

8. Yamaki, H. A conjecture of Frobenius and the sporadic simple groups II. Math. Comp. 1986, 46, 609-611.

9. Chen, K.; Meng, W.; Shi, J. On an inverse problem to Frobenius' theorem II. J. Algebra Appl. 2012, 11, 1250092.

10. Heineken, H.; Russo, F.G. Groups described by element numbers. Forum Math. 2015, 27, $1961-1977$. [CrossRef]

11. Heineken, H.; Russo, F.G. On a notion of breadth in the sense of Frobenius. J. Algebra 2015, 424, $208-221$. [CrossRef]

12. Heineken, H.; Russo, F.G. Classification of finite groups via their breadth. Glasgow Math. J. 2019. [CrossRef]

13. Meng, W.; Shi, J. On an inverse problem to Frobenius' theorem. Arch. Math. 2011, 96, 109-114. [CrossRef]

14. Meng, W. Finite groups of global breadth four in the sense of Frobenius. Commun. Algebra 2016, 45, 660-665. [CrossRef]

15. Meng, W.; Shi, J.; Zhang, C. On the Frobenius spectrum of a finite group. J. Algebra Appl. 2017, 16, 1750051.

16. Huppert, B. Endliche Gruppen, I; Springer: Berlin, Germany, 1967.

17. Zassenhaus, H. Über endliche Fastkörper. Abh. Math. Sem. Univ. Hamburg 1935, 11, 187-220. [CrossRef]

18. Herfort, W.; Hofmann, K.H.; Russo, F.G. Periodic Locally Compact Groups; de Gruyter: Berlin, Germany, 2018.

19. Hofmann, K.H.; Morris, S.A. The Structure of Compact Groups; de Gruyter: Berlin, Germany, 2013.

20. Klopsch, B.; Nikolov, N.; Voll, C. Lectures on Profinite Topics in Group Theory; Lectures from the Course on Asymptotic Methods in Infinite Group Theory Held in Oxford, September 2007, LMS 77; Cambridge University Press: Cambridge, UK, 2011.

21. Leedham-Green, C.R.; McKay, S. The Structure of Groups of Prime Power Order; Oxford University Press: Oxford, UK, 2002.

22. Lubotzky, A.; Segal, D. Subgroup Growth; Birkäuser: Berlin, Germany, 2003.

23. Ribes, L.; Zalesski, P. Profinite Groups; Springer: Berlin, Germany, 2000.

24. Wilson, J. Profinite Groups; Clarendon Press: Oxford, UK, 1998.

25. Lubotzky, A.; Mann, A. Powerful p-groups. I. Finite Groups. J. Algebra 1987, 105, 484-505. [CrossRef]

26. Lubotzky, A.; Mann, A. Powerful p-groups. II. p-adic analytic groups. J. Algebra 1987, 105, $506-515$. [CrossRef]

27. Sautoy, M.d.; Segal, D.; Shalev, A. New Horizons in Pro-p Groups; Progress in Mathematics 184; Birkhäuser: Boston, MA, USA, 2000.

28. Héthelyi, L.; Lévai, L. On elements of order p in powerful p-groups. J. Algebra 2003, 270, 1-6. [CrossRef]

29. Robinson, D. A Course in the Theory of Groups; Springer: Berlin, Germany, 1980.

30. Reid, C. The generalised pro-Fitting subgroup of a profinite group. Commun. Algebra 2013, 41, $294-308$. [CrossRef]

31. Reid, C. On the structure of just infinite profinite groups. J. Algebra 2010, 324, 2249-2261. [CrossRef]

32. Garonzi, M.; Patassini, M. Inequalities detecting structural properties of a finite group. Commun. Algebra 2017, 45, 677-687. [CrossRef] 
33. De Medts, T.; Tărnăuceanu, M. Finite groups determined by an inequality of the orders of their subgroups. Bull. Belgian. Math. Soc. Simon Stevin 2008, 15, 699-704. [CrossRef]

34. Tărnăuceanu, M. An arithmetic method of counting the subgroups of a finite abelian group. Bull. Math. Soc. Sci. Math. Roum. 2010, 53, 373-386.

35. Tărnăuceanu, M. A nilpotency criterion for finite groups. Acta Math. Hungarica 2018, 15, 499-501. [CrossRef]

(C) 2019 by the author. Licensee MDPI, Basel, Switzerland. This article is an open access article distributed under the terms and conditions of the Creative Commons Attribution (CC BY) license (http:/ / creativecommons.org/licenses/by/4.0/). 